\title{
In Situ TEM Investigation of Irradiation Induced Amorphization of Fe Oxide
}

\author{
Martin Owusu-Mensah, Jacob Cooper and Djamel Kaoumi
}

North Carolina State University, Raleigh, North Carolina, United States

Even though significant progress has been made in the past decades, there is still a lot of essential information that could contribute to a better understanding of material properties in extreme conditions. One typical area of limited information is the effect of radiation damage in oxides and the interfaces between metals and oxides. In fact, there is significant amount of research and data related to irradiation damage in steels but literature is scarce for radiation damage in oxides. Oxides grow at metal surfaces under the corrosive environment of nuclear reactors. Understanding the irradiation induced defect formation and their mobility in the oxides and across interfaces could provide valuable information in predicting the behavior of these materials at extreme conditions. There is reasonable information available in literature pertaining to the radiation damage in oxides with the fluoride[1], pyrochlore[1,2] or spinel crystal structures[3]. Surprisingly, there is very little information on the irradiation damage in various forms of oxides of iron and chromium systems even though these oxides remain the most common oxide formation in steels and thus in steel components in a reactor.

For that matter, Iron/iron oxide interfaces have been developed to investigate the irradiation damage in such metal-oxide interfaces by ion irradiation. In-situ ion irradiation technique within a TEM has shown to be a reliable technique for investigating the irradiation induced defects such as point defects, dislocations and stacking faults as it allows following directly the kinetics and mechanisms involved in the formation and evolution of these defects in real time with an enhanced spatial resolution down to the nanometer scale. In-situ ion irradiation of iron/iron oxide interfaces are hereby reported in this study focusing on the irradiation-induced defects and amorphization of after irradiation at low temperatures.

Iron/ Iron oxide interfaces: Pure iron oxides were naturally grown by Pacific Northwest National Laboratory (PNNL) on iron bars in air at temperatures of 600 and $400^{\circ} \mathrm{C}$ for a duration of 1 hour and 8 hours respectively. TEM specimens were prepared by Focused Ion Beam (FIB) lift-out technique using FEI Quanta 3D FIB system. The elemental composition characterization using ChemiSTEM elemental mapping and the crystallographic structure of the oxide interface by diffraction pattern in these as-received samples were determined to correspond to $\mathrm{Fe}_{3} \mathrm{O}_{4}$ with the $f c c$ crystal structure. These samples were irradiated at the Intermediate Voltage Electron Microscopy (IVEM) Tandem facility located at Argonne National Laboratory in the USA. Samples were irradiated using $1 \mathrm{MeV} \mathrm{Kr}^{2+}$ ions at cryogenic temperatures of 20 and $50 \mathrm{~K}$ up to doses of $15 \mathrm{dpa}$ in iron within a TEM. Samples were monitored during the entire irradiation process. The presence of defects of defects in the iron, iron oxide and the interface were carefully followed for each irradiation step where bright field images, dark field image and diffraction patterns were acquired at these irradiation steps for selected regions.

In the case of the irradiation at temperatures $50 \mathrm{~K}$, amorphous rings were observed in the diffraction pattern for iron oxide already at $1 \mathrm{dpa}$. These amorphous rings were present throughout the remaining irradiation steps until 15 dpa (as seen in Figure 1). In addition, loss of contrast of the oxide grains a feature of amorphization was also observed in certain grains confirming the possible amorphization of these oxide grains. However, not all the grains appear to amorphize at this temperature and a possible explanation could be due to the orientation of these oxide grains with respect to the irradiating ion beam. After allowing the sample to gradually warm back to room temperature, the diffraction pattern began showing Bragg 
spots along the amorphous ring suggesting a recrystallization of the sample after getting back to room temperature. Upon tilting the sample around, the oxide grains appeared to retain some form of contrast. This together with the recrystallization of the oxide grains probably suggest that the oxide grains did not fully amorphize. As expected, the metal on the other hand did not indicate any possibility of amorphization even until the maximum dose of $15 \mathrm{dpa}$. The amorphization of the iron oxide $\left(\mathrm{Fe}_{3} \mathrm{O}_{4}\right)$ grains describes the chemical and structure disordering under irradiation conditions. It is unclear what drives the amorphization of the oxide grains but the amorphization by Motta et al. [4] occurs by the local increase and accumulation of point defects. As a critical value is reached, the lattice becomes unstable and the crystalline or ordered structure may collapse leading to the amorphous structure. In relation to the amorphization resistance of oxides with the spinel structure such as that of $\mathrm{Fe}_{3} \mathrm{O}_{4}$, Uberuaga et al. [5] have demonstrated that the amorphization resistance of these oxides with the less-than fully dense cation sublattices directly correlates with the disordering energy or the ability to accumulate point defects. Such oxides are therefore more radiation tolerant than other oxides and probably difficult to amorphize completely. Elsewhere, for the irradiation performed at the $20 \mathrm{~K}$, the results were similar to that observed at $50 \mathrm{~K}$. Amorphization of the oxide appear to begin already at $0.5 \mathrm{dpa}$ with the presence of amorphous rings in the diffraction pattern. These rings as well as the loss of contrast of the oxide grains were observed until the maximum dose of $10 \mathrm{dpa}$. Again, upon allowing the sample to go back to room temperature, the possible recrystallization of the oxide grains was observed. Diffraction spots along the amorphous ring reappeared as observed for the irradiation at 50K. In the near future, HRTEM would be performed to confirm the crystal structure of suspected partial amorphization of the oxide.

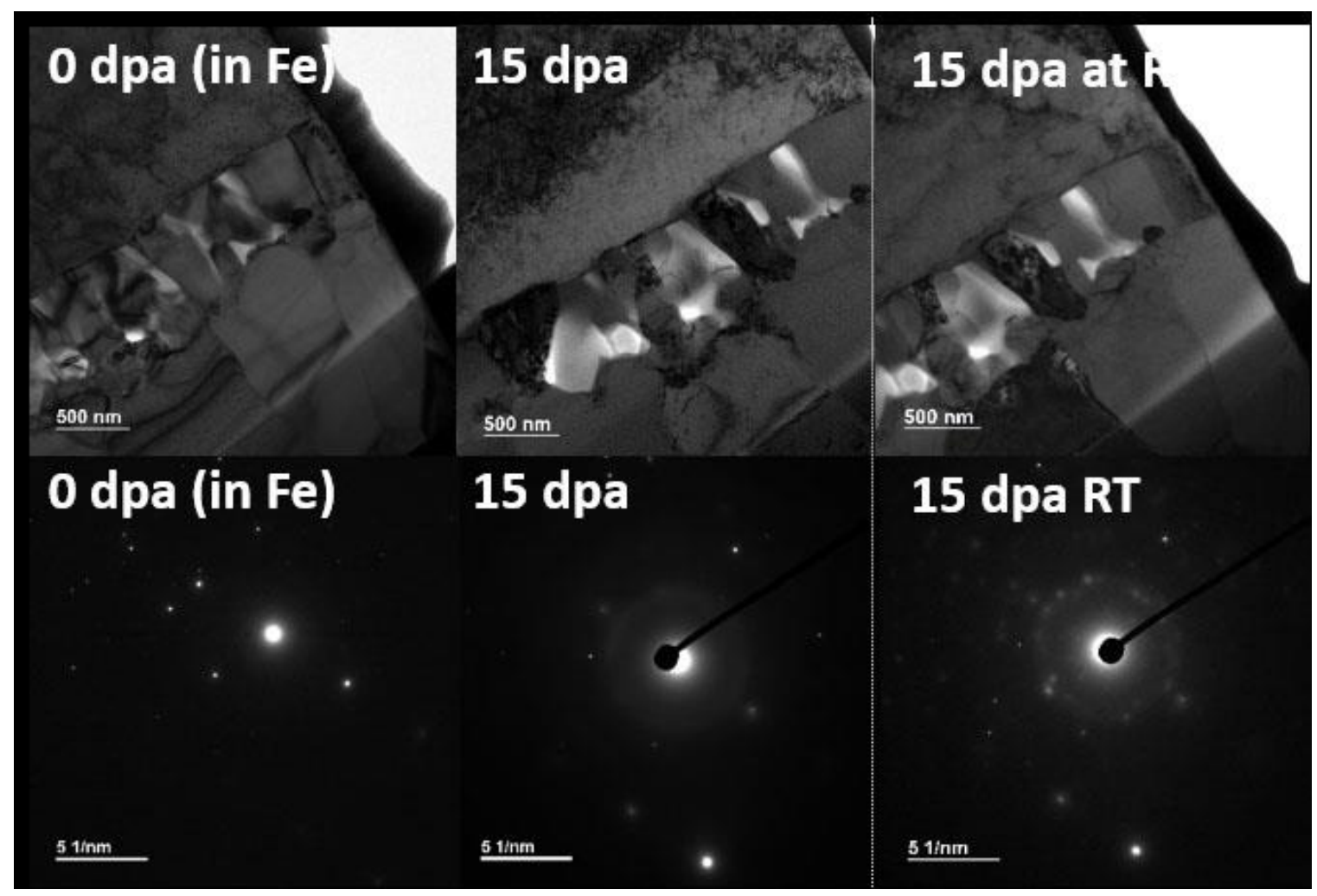

Figure 1. Bright Field images and diffraction patterns of the $\mathrm{Fe} / \mathrm{Fe}$ oxide interface irradiated at $50 \mathrm{~K}$ using $1 \mathrm{MeV} \mathrm{Kr}$ ions for irradiation doses ranging from 0 to $15 \mathrm{dpa}$ for $50 \mathrm{~K}$. The sample was subsequently left to get back to room temperature and the diffraction pattern obtained is shown. 


\section{References}

[1] A. K. E. Sickafus et al., "Radiation Tolerance of Complex Oxides Published by: American Association for the Advancement of Science," vol. 289, no. 5480, pp. 748-751, 2000.

[2] G. R. Lumpkin et al., "Nature of the chemical bond and prediction of radiation tolerance in pyrochlore and defect fluorite compounds," Journal of Solid State Chemistry, vol. 180, pp. 1512-1518, 2007.

[3] K. E. Sickafus et al., "Cation disorder in high dose, neutron-irradiated spinel," Journal of Nuclear Materials vol. 219, pp. 128-134, 1995.

[4] A. T. Motta, "Amorphization of intermetallic compounds under irradiation - A review," Journal of Nuclear Materials vol. 244, pp. 227-250, 1997.

[5] B. P. Uberuaga et al., "Opposte correlations between cation disordering and amorphization resistance in spinels versus pyrochlores," Nature Communications, vol. 6, pp. 1-8, 2015. 